\title{
Analytic Hepatic Deterioration in Rectal Cancer: Liver Steatosis Vs Disease Progression
}

\author{
Losada Vila Beatriz* \\ Medical Oncologists in Fuenlabrada Hospital, Madrid, Spain \\ *Corresponding author: Losada Vila Beatriz, Medical Oncologists in Fuenlabrada Hospital, Madrid, Spain. \\ To Cite This Article: Losada Vila Beatriz. Analytic Hepatic Deterioration in Rectal Cancer: Liver Steatosis Vs Disease Progression. Am J Biomed \\ Sci \& Res. 2019 - 4(2). AJBSR.MS.ID.000770. DOI: 10.34297/AJBSR.2019.04.000770
}

Received: July 05, 2019 | Published: July 22, 2019

\section{Introduction}

Hepatic steatosis is a phenomenon that is often objectified in imaging tests that, if there is no analytical alteration of the hepatic profile, does not need further follow-up. It can be caused by chemotherapy treatment, so in oncological patients should be monitored more closely. We present the case of a patient treated with capecitabine who, once the treatment was finished, began to increase bilirubin and transaminases, showing an increased diffuse hepatic steatosis in an MRI.

\section{Clinical Case}

A man of 65 years old diagnosed of rectal adenocarcinoma, started chemoradiation (capecitabine) in July 2018. Good tolerance of treatment. After finishing treatment, bilirubin started to elevate (2.4), without fever or clinical infection. Surveillance at next week was performed, showing progressive elevation of $\mathrm{Br}$, without use of new drugs and more than 1 month without treatment of capecitabine. Hepatitis serologies showed no reactivation of hepatitis. Also, CT was performed, with disease in response and steatosis increasing compared to previous CT. No cholelithiasis in CT. He was referred to Emergency for clinical assessment. He refers increase of consumption of eggs and beer (not daily). Valerate by Digestive Team, he started on steroids, improving bilirubin levels (normal at 4 days) and restricting fat in meals. No liver biopsy was needed. Finally, he continued revisions, he was operated and no disease recurrence.

\section{Discussion}

Chemotherapy treatment can worsen the liver profile, requiring dose adjustment or rest of the treatment. However, in oncological patients another aspect to rule out is that it is altered by a progression at the liver level.

In this patient, treated with a radical intention and without liver disease at diagnosis, the first thing to think, since the hepatic profile had not been elevated during the treatment but at the end, was that it could have presented a reactivation of the hepatitis virus.
However, this was ruled out. Once the differential diagnosis was made, an image test was repeated, showing an increase in hepatic steatosis that was present prior to the diagnosis. The treatment is corticotherapy and from the Digestive hepatic biopsy was proposed. The risk of steatohepatitis with capecitabine is not known. In other drugs like tamoxifen, it has been more studied. The first month of tamoxifen treatment induced more changes in the biochemical parameters analyzed, particularly in serum lipid profiles and liver enzymes levels, when compared with the second or third months of treatment. All the biochemical changes quantified suggest that the dose of tamoxifen used could induce functional alterations during the first month of tamoxifen treatment, but that, afterwards induced adaptive changes [1]. So chronological risk is not well known even for the most common drugs.

The present hematologic data corroborate the histopathological findings, since the percentage of hepatocytes containing small lipid vacuoles was classified as moderate steatosis only in the first month of TAM treatment and was reduced upon two and three months of tamoxifen treatment. In addition, the present study showed that tamoxifen treatment could induce changes in glycogen storage and in glucose serum levels, suggesting time-dependent effects in glucose metabolism. Recently it was shown that tamoxifen can reduce adipose tissue accumulation by inducing reactive oxygen species production. In the liver, tamoxifen seems to be able to promote the inhibition of gluconeogenesis, the stimulation of glycolysis and the deposition of fatty liver [2]. While generally well tolerated, one of the most common side effects of tamoxifen is the development of nonalcoholic fatty liver disease (NAFLD), which is characterized by abnormal accumulation of triglycerides in liver. This disease includes a wide spectrum of malady from simple steatosis through steatohepatitis, cirrhosis and hepatocellular carcinoma [3].

Nevertheless, tamoxifen-associated steatohepatitis seems to occur mostly in obese people [4], suggesting that tamoxifen in combination with obesity could lead to further progression of 
steatosis. Indeed, obesity is also associated with hepatic steatosis due to an abnormal mitochondrial function, suggesting that tamoxifen could worsen the pre-existing mitochondrial dysfunction present and, that this combination could lead to increased oxidative stress, cytokine induction, and steatohepatitis lesions [4].

\section{Conclusion}

Worsening of liver function in oncological patients is usually related with hepatitis reactivation, disease progression and sometimes liver steatosis Chronological risk of appearing steatohepatitis is not well known even for the most common drugs. Obesity and alcohol are two factors associated with liver dysfunction Sometimes treatment requires steroids and liver biopsy.

\section{References}

1. Begriche K, Massart J, Robin MA, Borgne-Sanchez A, Fromenty B (2011) Drug-induced toxicity on mitochondria and lipid metabolism: mechanistic diversity and deleterious consequences for the liver J Hepatol 54: 773-794.

2. Bruno S, Maisonneuve P, Castellana P, Rotmensz N, Rossi S, et al. (2005) Veronesi Incidence and risk factors for non-alcoholic steatohepatitis: prospective study of 5408 women enrolled in Italian tamoxifen chemoprevention trial. BMJ 330: 932.

3. Begriche K, Massart J, Robin MA, Bonnet F, Fromenty B (2013) Mitochondrial adaptations and dysfunctions in nonalcoholic fatty liver disease. Hepatology 58: 1497-1507.

4. Leal S, Rocha L, Silva A, faria J, Dinis-Oliveira RJ, et al. (2018) Evaluation of progressive hepatic histopathology in long-term tamoxifen therapy. Pathol Res Pract 214: 2115-2120. 